\title{
CONNECTIONS BETWEEN OBJECT CLASSIFICATION CRITERIA USING AN ULTRASONIC BI-SONAR SYSTEM
}

\author{
BOGDAN KRECZMER ${ }^{a}$ \\ ${ }^{a}$ Chair of Cybernetics and Robotics \\ Wrocław University of Technology, Wybrzeże Wyspiańskiego 27, 50-320 Wrocław, Poland \\ e-mail: bogdan. kreczmer@pwr . edu.pl
}

\begin{abstract}
The paper presents connections between the criteria which make three types of objects possible to be recognized, namely, edges, planes and corners. These criteria can be applied while a binaural sonar system is used. It is shown that the criteria are specific forms of a general equation. The form of the equation depends on a single coefficient. In the paper, the meaning of this coefficient is discussed. The constructions of the arrangement of objects are presented and are bound with values of the coefficient.
\end{abstract}

Keywords: ultrasonic range-finder, multi-reflection, bi-sonar system.

\section{Introduction}

The ultrasonic range finders which are used in robotics usually work at a frequency of $40 \mathrm{kHz}$ (piezoelectric transducers) or $50 \mathrm{kHz}$ (electrostatic transducers). Since irregularities of object surfaces in indoor environments are much smaller than the wavelength of the signal (about $9 \mathrm{~mm}$ for $40 \mathrm{kHz}$ and $7 \mathrm{~mm}$ for $50 \mathrm{kHz}$ ), an echo is produced by specular reflection. In this way, acoustic energy is reflected analogously to a mirror reflecting light (Brown, 1985; Kuc and Siegel, 1987). This fact is applied effectively in many methods of object detection (Barshan and Kuc, 1990; Peremans et al., 1993; Kleeman and Kuc, 1994; Heale and Kleeman, 2001; 2002). These methods also make it possible to identify some of the objects. The well-known classification standard for two-dimensional indoor target types is that of planes, corners and edges (Bozma and Kuc, 1991; Barshan and Kuc, 1990; Heale and Kleeman, 2001; Kleeman and Kuc, 1994; Leonard and Durrant-Whyte, 1991; Peremans et al., 1993).

In the work of Kleeman and Kuc (1994), these targets were defined as follows: a plane is assumed to be a vertical smooth flat surface that reflects ultrasound specularly; a corner is a concave intersection of two planes at a right angle; an edge is assumed to reflect ultrasound from a point that is approximately independent of the transmitter and receiver position. To distinguish the targets, methods based on measurements of the time of flight (TOF) can be applied (Peremans et al., 1993; Heale and Kleeman, 2001; Kleeman, 2002). They are the most effective. To discriminate between targets, a separate criterion is constructed for an edge, a plane and a corner (Heale and Kleeman, 2001). Hence, a set of three criteria was collected. They were treated as independent and unbound equations. In the work of Kreczmer (2010), it was shown that they are specific forms of a more general criterion. This criterion contains an additional coefficient $\rho$. Its value determines a specific form of the criterion. While the coefficient has the value -1 , a criterion for corners is obtained. The criteria for edges or planes are produced if $\rho$ is equal to 0 or 1 , respectively.

In this situation, some natural questions arise: What is the meaning of the coefficient $\rho$ ? Does it have a physical interpretation? Is it combined with any feature of an object? Can it be represented as a continuous and smooth map of this feature? What does an object look like with this feature for which $\rho$ is equal to, e.g., 0.5 or a different value?

This paper addresses the problems stated in the questions above and gives the answers. The main idea of the approach to these problems is presented by Kreczmer (2013). This paper extends the study regarding these problems. It also contains some experimental results that demonstrate the important properties of ultrasonic signal propagation, which are exploited in the presented approach. These results confirm that the main concept of 
the approach is a good one.

The problem discussed in this paper is presented in the context of ultrasonic detection but, in fact, it is a more general one. It also refers to radars or other devices whose measurements are based on reflection of an electromagnetic wave. It is worth noting that even by using Wi-Fi routers it is possible to effectively exploit the reflection effect (Adib and Katabi, 2013; Nanani and Prasad, 2013).

The paper is organized as follows. The next section introduces related works. In Section 3, forms of identification criteria are discussed. Section 4 describes constructions which make it possible to transit smoothly from the case of a corner to the case of an edge, and then to the case of a plane. Section 5 presents equations which combine the coefficient $\rho$ with parameters that represent some features of the proposed constructions. Section 6 gives the conclusions and answers to the questions stated in the introduction.

\section{Related work}

A simple ultrasonic range finder only delivers information about the TOF, which is used for the approximation of the distance of flight (DOF) and, finally, the distance to an object. Using a more advanced sonar system, it is also possible to get information about the magnitude of the echo amplitude and the inclination angle of the arrival direction of an echo. In the work of Barshan and Kuc (1990), it was shown that, to distinguish edges, corners and planes, it is sufficient to compare the amplitude of an echo registered by two receivers. The method requires separate signal emission performed by two transmitters and two measurements of echo amplitudes registered by both receivers. Edges, planes and corners can be discriminated by testing the angle difference between the echo bearings obtained after a separate signal emission performed by two transmitters (Kleeman and Kuc, 1994; 1995). The sonar system used in that approach consists of three transducers. Two of them are transceivers (this type of transducer acts first as a transmitter and then as a receiver). The third transducer is a receiver only.

In the work of Peremans et al. (1993), a method based on TOF measurement was presented. The sonar system, which is exploited in this approach, also consists of three transducers (a single transmitter and two receivers). The proposed method makes it possible to discriminate between edges and planes only. It is not possible to distinguish planes and corners. To discriminate between them, a movement of such a sensor system is required. While the position is not changed, one transmitter and any number of receivers are insufficient to distinguish the targets in any orientation (Kleeman and Kuc, 1994; 1995). It was established that two transmitters and two receivers are necessary and sufficient

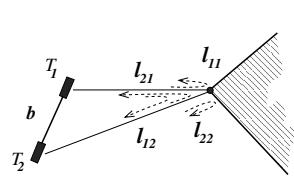

(a)

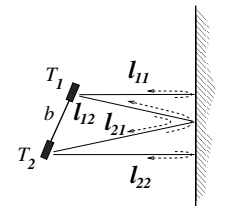

(b)

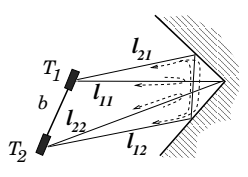

(c)
Fig. 1. Sketch of signal paths for an edge (a), a plane (b), a corner (c).

for discriminating between planes, corners and edges in two dimensions (Kleeman and Kuc, 1994; 1995).

A much simpler set of criteria than that mentioned above is presented by Heale and Kleeman (2001) as well as Kleeman (2002). It consists of three independent equations and each of them is combined with a single type of target (i.e., edges, planes or corners). These criteria are treated in this paper as unrelated ones. The system presented by Heale and Kleeman (2001) exploits a DSP echo processor. It makes it possible to obtain a repetition rate of about $27 \mathrm{~Hz}$. Because the measurements and data processing are performed fast, it is possible to classify targets on-the-fly. Its reliability can be increased by taking into account robot speed (Kleeman, 2004). The sonar system used in the discussed approach consists of four transducers, namely, two transmitters, and two receivers. When replacing them with two transceivers a minimal configuration of the sonar system is obtained, which allows all three targets to be distinguished. The efficiency of their discrimination strongly depends on the accuracy of DOF measurement. The more accurate the measurements, the more reliable the discrimination of the targets. It also makes it possible for the sonar system to be more compact (transducers can be placed closer to each other). In the papers presented above, an accuracy of about $1 \mathrm{~mm}$ in still air is reported. Queirós et al. (2010) show that it can be increased by up to $0.1 \mathrm{~mm}$. A review of the current state-of-the-art acoustic airborne ranging methods is presented by Jackson et al. (2013).

\section{Identification criteria}

The optimal configuration of a sonar system, which makes it possible to distinguish edges, planes and corners, consists of two sonars. Each of them should work as a transmitter and also as a receiver. The method requires the application of two measurements. During each of them, one of the two sonars is used as a transmitter. After signal emission, both sonars are switched into receiving mode. Then, each of them separately registers the time of the echo flight. A schema of measured signal paths for all cases is presented in Fig. 1. The relationship of distance paths is much better to analyze when an approach based on an image of a virtual sonar system is applied for 
the case of the plane and the corner (see Fig. 2(b),(c)) (Barshan and Kuc, 1990; Kleeman and Kuc, 1995). An analogous approach can be employed in the case of the edge (see Fig. 2(a)). It simplifies the geometrical analysis. For the case presented in Fig. 22a), it is not difficult to show that

$$
\text { edge } \quad \rightarrow \quad l_{12}+l_{21}-l_{11}-l_{22}=0 .
$$

The equations figured out for a plane and a corner are as follows:

$$
\begin{gathered}
\text { plane } \rightarrow \quad\left(l_{12}+l_{21}\right)^{2}-4 b^{2}-4 l_{11} l_{22}=0, \\
\text { corner } \rightarrow \quad\left(l_{12}+l_{21}\right)^{2}+4 b^{2}-2 l_{11}^{2}-2 l_{22}^{2}=0 .
\end{gathered}
$$

It seems that the three equations above have nothing in common. This impression changes when the last equation is rewritten in an equivalent form. To work out this form, it is necessary to notice that $l_{11}, l_{22}, l_{12}, l_{21}>0$. Taking this into account, the equivalent form is

$$
\text { edge } \rightarrow \quad\left(l_{12}+l_{21}\right)^{2}-\left(l_{11}+l_{22}\right)^{2}=0 .
$$

Now, it is straightforward to figure out that the criteria (1) and (2) are special cases of the same equation,

$$
\left(l_{12}+l_{21}\right)^{2}-\left(l_{11}+l_{22}\right)^{2}+\rho\left(\left(l_{11}-l_{22}\right)^{2}-4 b^{2}\right)=0 .
$$

The criterion for an edge can be obtained while $\rho=0$. The criterion for a plane is acquired while $\rho=1$. Putting $\rho=-1$, Eqn. (3) is transformed into the criterion for a corner. This form of that equation was presented by Kreczmer (2010). When considering the coefficient $\rho$, some questions arise about its meaning. They are stated in Section 1.

Figure 1 shows all the standard cases which can be identified using ultrasonic systems. Presentation of them in this way can be found in many other papers (Bozma and Kuc, 1991; Kleeman and Kuc, 1994; Heale and Kleeman, 2001). It seems to suggest that the value of the coefficient $\rho$ represents convexity or non-convexity of an object. Unfortunately, this is an erroneous conclusion. To prove this, we can notice that the smooth transformation of an object, which goes through these cases, should start from an edge-like object, or from a corner. When starting from an edge-like object the transformation should allow us to change the object step by step into a plane, and finally into a corner. Nonetheless, the subsequent values of $\rho$ are not consistent with this line of reasoning. They do not change smoothly from -1 to 1 or from 1 to -1 . At the beginning of the transformation, the value is 0 , because we start from an edge-like object. When gradually changing it into the plane, the coefficient $\rho$ reaches the value 1 . Continuing this process and changing the plane into a corner, the value -1 is finally obtained. That notwithstanding, the assumption that an object is a

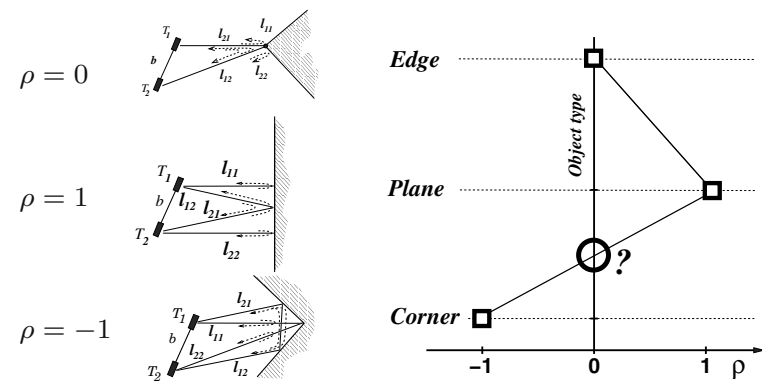

Fig. 3. Sketch of the transition path from a corner to a plane and then to an edge.

function of the value $\rho$ fails (see Fig. 3), because when transforming a plane into a corner $\rho$ is swept from 1 to -1 . Thus, the value 0 is again reached. This means that another object should exist with $\rho=0$.

The wrong suggestion that $\rho$ represents convexity or non-convexity of an object is caused by the image of the first object in Fig. 1 The flight time of echoes produced by an edge does not depend on the arrangement of planes, whose connection creates the edge, as long as this part of the object is convex. The same final results of measurements can be obtained for a rod. In that way, it gives a more realistic picture of this phenomenon (see Fig. (4). When considering the values of the parameter $\rho$ for the presented cases, it can be presumed that a kind of transition between the objects shown in Fig. 4 exists. Thus the problem consists in finding such an object construction which can be parameterized by $\rho$. For certain values, it should be possible to obtain a corner, a rod and finally a

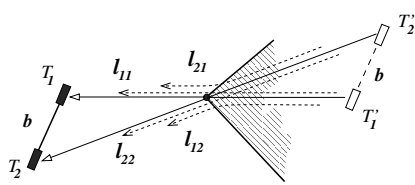

(a)

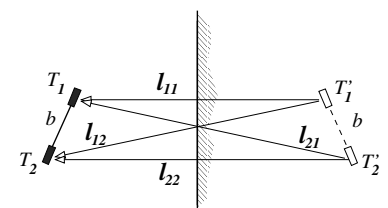

(b)

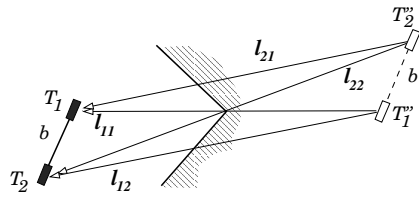

(c)

Fig. 2. Sketch of signal paths by applying the approach of the image of a virtual two-transducer system for an edge (a), a plane (b), a corner (c). 


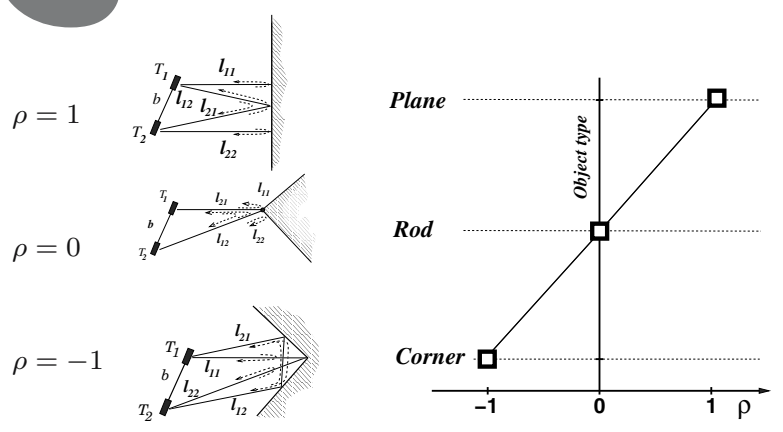

Fig. 4. Sketch of the transition path from a corner to a rod and then to a plane.

plane. Transition forms of an object should be combined with values of $\rho$ that are different than $-1,0$ and 1 .

\section{Construction of transition objects}

Considering transition between the objects of interest, it is possible to distinguish two cases which seem to be substantially different. The first one is when the form of the object starts as the corner and ends as the rod. The second case is an analogical transition for the object which starts as the rod and ends as the plane. Therefore, further on in this paper, both the cases are discussed separately. To make analysis more straightforward, it is assumed that the sonar system is located in a way that $l_{11}=l_{22}$ (see Fig. 5).

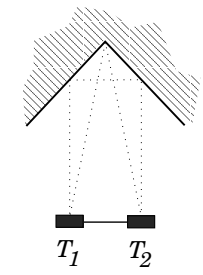

(a)

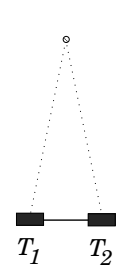

(b)

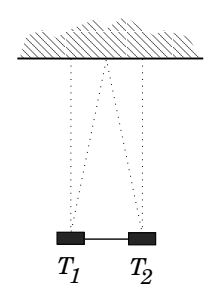

(c)
Fig. 5. Location of the sonar system in relation to a corner (a), a rod (b), a plane (c). For each case, $l_{11}=l_{22}$.

\subsection{Construction of transition objects for the rod and} the plane. When analyzing Fig. 5(b) and (c), it can be noticed that the signal paths $l_{12}$ and $l_{21}$ are the same in both the figures. Thus considering all object forms obtained by its successive transformations, a reasonable assumption is that these paths should not be changed for all these forms, either. This assumption is met when the result of transformation is a cylinder. If the radius of the cylinder is close to zero, then the rod is obtained. When increasing the radius, a plane is created by a part of the cylinder's surface, while the value of the radius goes to infinity (see Fig. 6).

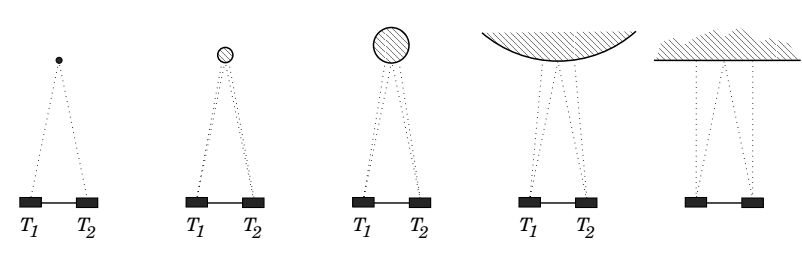

Fig. 6. Transition from the rod to the plane using cylinders.

4.2. Construction of transition objects for the rod and the corner. Considering Fig. 5(a) and (b), one can notice that $l_{11}$ and $l_{22}$ are the same in both cases. This feature is very characteristic, especially for the corner because it creates a special specular reflector. A signal emitted towards the corner is reflected back, exactly towards the source of emission. This is because of the junction of two perpendicular planes. Such a junction is responsible for producing a double reflection which has the property described above.

Following the same line of arguments exploited in the previous section, we can infer that this feature is preserved for all transitional objects. Thus the objects have to have a construction similar to the kind of special reflector discussed above. This can be obtained when cylinders are used again in order to approximate each plane. To create a corner-like reflector, the cylinders have to cross each other along the same edge by which the planes of a corner are joined. Moreover, at that place, the surfaces of the cylinders have to be perpendicular to each other. When applying the same approach, which is presented in the previous section, the rod-like object is obtained while the radii of the cylinders are decreased to zero (see Fig. 7). The corner is created while their radii go to infinity.

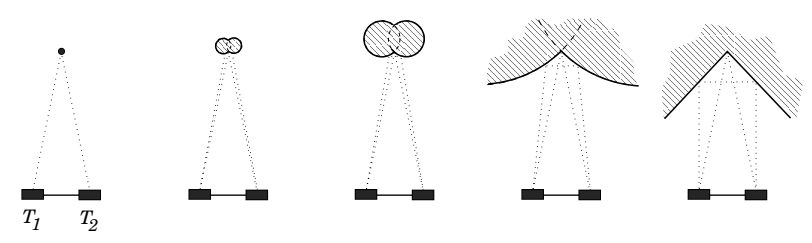

Fig. 7. Transition from the rod to the corner using cylinders.

This construction has an important disadvantage. Considering the case presented in Fig. 8 (a), it can be noticed that it is possible to find a straight path from a transducer, e.g., $T_{1}$, to the closest cylinder in a way that the path is perpendicular to the surface of the cylinder. In Fig. 8, a), this path is denoted as $l_{11}^{\prime}$. Unfortunately, the path is shorter than $l_{11}$. This means that the first echo comes along path $l_{11}^{\prime}$. While a sent wave train of a signal is sufficiently long, it is not possible to register the second echo which comes along $l_{11}$. Nevertheless, these are not new phenomena. It is possible to find the same case for the corner (see Fig. 8 (b)). Then, the question arises: Why 


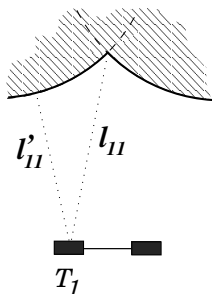

(a)

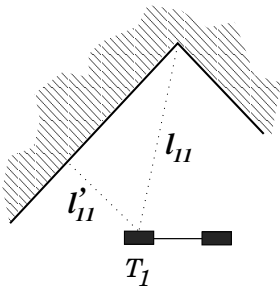

(b)
Fig. 8. Sketch of signal paths for two cylinders (a), a corner (b). For both cases, the path $l_{11}^{\prime}$ is shorter than $l_{11}$.

is it possible to measure the distance $l_{11}$ for the corner at all? Using standard ultrasonic range finders, the first echo is only registered. Thus, the wave train should be detected which passes along $l_{11}^{\prime}$ instead of $l_{11}$.

This is caused by the physical features of ultrasonic transducers. In general, the signal is more attenuated if the direction of signal propagation is further away from the acoustic axis of a transducer. The same can be said of the amplitude of a received echo. This feature means that an object can be detected by a sonar if it is located in a certain cone built around the sonar's acoustic axis. Thus, when $l_{11}^{\prime}$ is outside the cone, the echo traveling along this path cannot be detected. Therefore, in such a case the distance $l_{11}$ is measured instead of $l_{11}^{\prime}$.

The shape of signal paths presented for a single cylinder does not cause any doubt. The assumptions of the shape of the path were applied by Peremans et al. (1991; 1993) or Barshan (1999), and correct results were obtained. Moreover, the method proposed by Peremans et al. (1993) was used for mobile robot navigation (Rencken et al., 1994; Möller, 1995). Hence, the transition between a rod and a plane is well defined.

It is not possible to say the same about the transition between a rod and a corner. It is not clear that two cylinder surfaces, which are connected at a right angle, cause a sufficiently strong double reflection to be noticed. Considering two planes connected at a right angle, the effect of a double reflection is clearly observed. It is sufficient to use a simple rotating ultrasonic range finder (see Fig.9(a)). When scanning a sector around the connection line of two planes, measurements of the distance are obtained, which suggest the presence of a single plane in that spot (see Fig. 9(b)). This phenomenon is well known (Kuc and Siegel, 1987; Barshan and Kuc, 1990; Bozma and Kuc, 1991; Leonard and Durrant-Whyte, 1991; 1992).

For two cylindrical surfaces, which are connected at a right angle (see Fig.10(a)), it is assumed that the same phenomena should be observed. Because the surfaces of the object are convex, it can be expected that the area where these surfaces will be detected should be closer to the place of the connection of both cylinder faces.

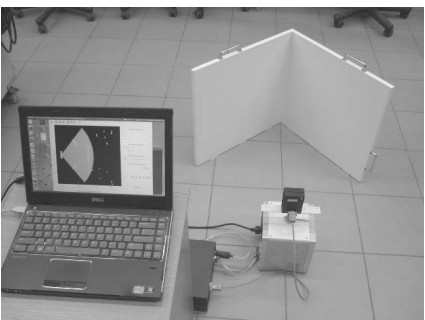

(a)

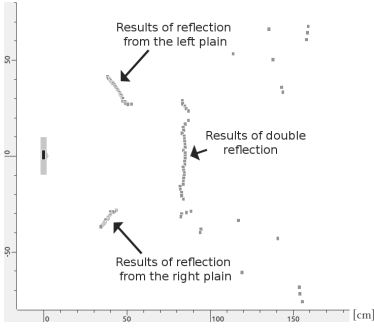

(b)
Fig. 9. Corner and its ultrasonic image of distance measurements: the laboratory stand for obtaining a sonar scan of distances to the corner-like object (a), results of distance measurements acquired by an ultrasonic range finder which scanned its environment at every $1^{\circ}(\mathrm{b})$.

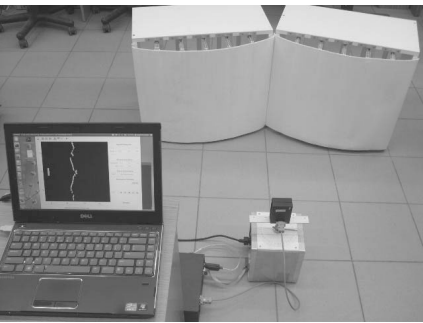

(a)

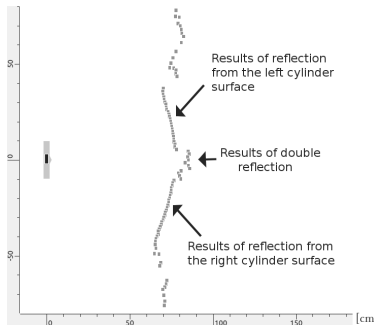

(b)
Fig. 10. Two cylinder surfaces connected at a right angle and their ultrasonic image of distance measurements: the laboratory stand for obtaining a sonar scan of distances to the object (a), results of distance measurements acquired by an ultrasonic range finder which scanned its environment every at $1^{\circ}$ (a). The radius of curvature of each surface is $2 \mathrm{~m}$.

Thus, the region in which double reflection is observed should be smaller. The obtained results (see Fig. 10) meet the expectations. This proves that the presented transition between a rod and a corner is properly constructed.

In summary, the presented construction of transitional forms does not introduce any new phenomena. It causes only that $l_{11}^{\prime}$ is measured instead of $l_{11}$, while the radii of the cylinders are sufficiently small. Proper measurement of $l_{11}$ for small radii is a technical matter of the extraction of a second echo.

\section{Equations for transitional forms}

Assuming that the arrangements of objects and the ultrasonic sonar system are the same as presented in Fig. 5allows the necessary computations to be simplified. Because in this case $l_{11}=l_{22}$, the general equation (3) can be transformed into the form

$$
\rho=\frac{1}{4 b^{2}}\left(\left(l_{12}+l_{21}\right)^{2}-\left(l_{11}+l_{22}\right)^{2}\right) .
$$


5.1. Transition from the plane to the rod. Considering the signal paths presented in Fig. 11, it is simple to show that the lengths of the paths can be expressed by the forms

$$
\begin{aligned}
& l_{12}=l_{21}=2 \sqrt{\frac{b^{2}}{4}+d^{2}}, \\
& l_{11}=l_{22}=2\left(\sqrt{\frac{b^{2}}{4}+(d+r)^{2}}-r\right) .
\end{aligned}
$$

This makes it possible to rewrite (4) and express $\rho$ as follows:

$$
\rho=\frac{4}{b^{2}}\left(\frac{b^{2}}{4}+d^{2}-\left((d+r) \sqrt{\frac{b^{2}}{4(d+r)^{2}}+1}-r\right)^{2}\right) .
$$

The form (5) shows that $\rho$ is a function in terms of $b$ - the distance between sonars, $d$ - the distance from the sonar system to the cylinder, and $r$-the radius of the cylinder. Let us denote this function as $\rho_{r-p}(r, d, b)$. It is straightforward to show that $\rho_{r-p}(0, d, b)=0$ for $d, b>0$. Moreover, it can be noticed that

$$
\begin{aligned}
& \lim _{r \rightarrow \infty} \rho_{r-p}(r, d, b) \\
& =\lim _{r \rightarrow \infty} \frac{4}{b^{2}}\left(\frac{b^{2}}{4}+d^{2}-\left((d+r) \frac{b^{2}}{8(d+r)^{2}}+d\right)^{2}\right)=1 .
\end{aligned}
$$

This meets the previous assumption that, for $r=0$, the cylinder is shrunken to the rod. As $r \rightarrow \infty$, the part of the cylinder face near the sonar system can be treated as a plane.

The values of $\rho_{r-p}(\cdot)$ depend on the distance $d$ to the object and the distance $b$ between sonars. The influence of $d$ on the values of $\rho_{r-p}(\cdot)$ is noticeable. Figure 12 shows the charts of $\rho_{r-p}(\cdot)$ computed for $b=0.1 \mathrm{~m}$ and three values of $d$, namely, $0.5 \mathrm{~m}, 1 \mathrm{~m}$ and $2 \mathrm{~m}$. The parameter $b$ is less important. Its influence on $\rho_{r-p}(\cdot)$ is very small. Using the same scale in Fig. 13 as is used in Fig. 12 makes it impossible to distinguish the charts of $\rho_{r-p}(\cdot)$ drawn for $b=0.05 \mathrm{~m}$ and $0.25 \mathrm{~m}$.

5.2. Transition from the corner to the rod. The case of the transitional forms for the transition from the corner

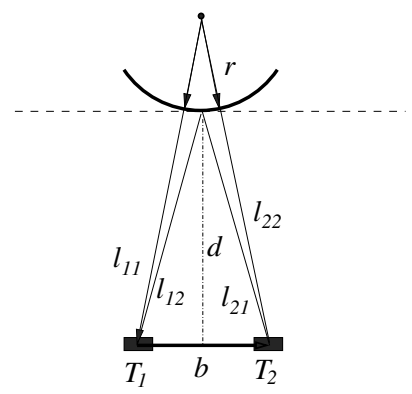

Fig. 11. Signal paths for a single cylinder.

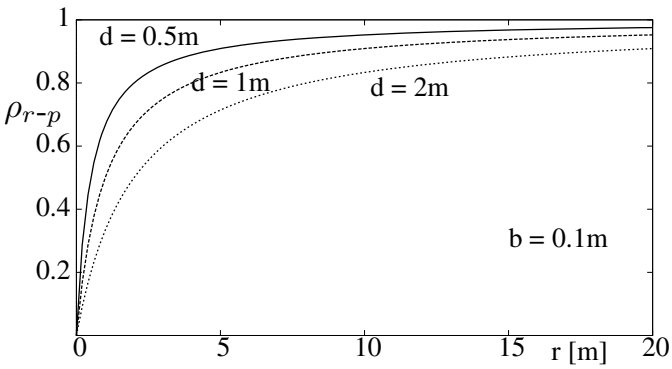

Fig. 12. Graph of the function $\rho_{r-p}(r, d, b)$ for $b=0.1 \mathrm{~m}$ and $d=0.5 \mathrm{~m}, 1 \mathrm{~m}$, and $2 \mathrm{~m}$.

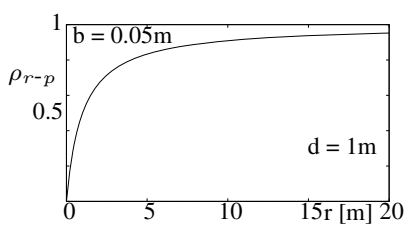

(a)

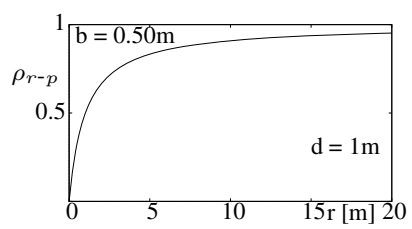

(b)
Fig. 13. Graph of the function $\rho_{r-p}(r, d, b)$ for $d=1 \mathrm{~m}$ and $b=0.05 \mathrm{~m} \mathrm{(a),} b=0.25 \mathrm{~m}$ (b).

to the rod is a bit more complicated. However, the lengths of the signal paths $l_{11}$ and $l_{22}$ (see Fig. 14) are trivial to determine and are

$$
l_{11}=l_{22}=2 \sqrt{\frac{b^{2}}{4}+d^{2}} .
$$

Computation of $l_{12}$ and $l_{21}$ is more complicated because

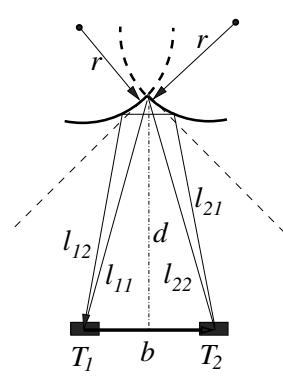

Fig. 14. Paths of a signal for cylinders which create a cornerlike object.

at first it is necessary to determine the locations of points where a signal is reflected (see Fig. 15). To this end, let us assume that the origin of the global coordinate system is in the place where both the cylinders are crossed (see Fig. 15). The lengths of both paths $l_{12}$ and $l_{21}$ are the same. Moreover, the arrangement of all paths is symmetrical with respect to the axis $O Y$. Taking this into account, it is convenient to divide the half part of path $l_{12}$ into two line sections $l_{a}$ and $l_{b}$ (see Fig. 15 a)). This gives 


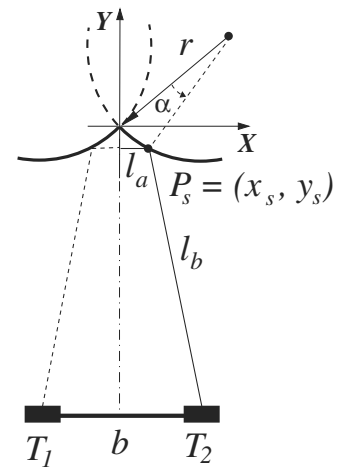

(a)

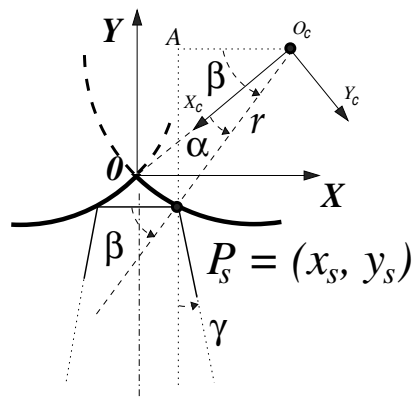

(b)
Fig. 15. Location of points of signal reflection: a signal path (a), the angle $\gamma(b)$

the simple equation

$$
l_{12}=l_{21}=2\left(l_{a}+l_{b}\right) .
$$

Section $l_{a}$ starts from the axis $O Y$ and ends at point $P_{s}$. The next section $l_{b}$ starts from $P_{s}$ and ends at $T_{2}$. Thus, the lengths of the sections are

$$
l_{a}=x_{s}, \quad l_{b}=\sqrt{\left(x_{s}-\frac{b}{2}\right)^{2}+\left(y_{s}-d\right)^{2}} .
$$

In the next step, $x_{s}$ and $y_{s}$ should be determined as functions of the cylinder radius. Having that, it will be possible to express $l_{12}$ and $l_{21}$ as a function of $r$ because of (8) and (7). In consequence, it will be possible to express $\rho$ as a function of $r$.

In a natural way, the coordinates of point $P_{s}$ can be expressed in the local coordinate system of the right cylinder. Assuming that the origin of this system is placed in the center of the cylinder and its axis $O_{c} X_{c}$ goes through the origin of the global coordinate frame (see Fig. 15(b)), the Cartesian coordinates of point $P_{s}$ are $(r \cos \alpha, r \sin \alpha)$. Applying a simple transformation, the coordinates of point $P_{s}$ in the global frame are

$$
\left\{\begin{array}{l}
x_{s}=\frac{r}{\sqrt{2}}(1+\sin \alpha-\cos \alpha), \\
y_{s}=\frac{r}{\sqrt{2}}(1-\sin \alpha-\cos \alpha) .
\end{array}\right.
$$

We are going to get formulae which will express $x_{s}$ and $y_{s}$ in terms of the parameters $r, b$ and $d$. To eliminate $\alpha$, a relation is useful which combines the angle $\gamma$ (see Fig. 15(b)) and the coordinates $x_{s}$ and $y_{s}$, namely,

$$
\operatorname{tg} \gamma=\frac{\frac{b}{2}-x_{s}}{d+y_{s}}
$$

Keeping in mind that the object surfaces considered are smooth, the angle of incidence of the signal is equal to the angle of its reflection. This makes it possible to establish the relation between $\alpha$ and $\beta$ (see Fig. 15(b)) as follows:

$$
\gamma=2 \beta-\frac{\pi}{2}=2 \alpha
$$

Applying this relation and using Eqns. (9) and (10), we get

$$
\frac{\sin 2 \alpha}{\cos 2 \alpha}=\frac{\frac{b}{2}-\frac{r}{\sqrt{2}}(1+\sin \alpha-\cos \alpha)}{d+\frac{r}{\sqrt{2}}(1-\sin \alpha-\cos \alpha)} .
$$

Unfortunately, transformation of this formula in order to express $\alpha$ as a function of $r$ using elementary functions does not seem possible. However, we can estimate the values of $\alpha$ for the whole range of $r$. Note that, as $r \rightarrow \infty$, the angle $\alpha \rightarrow 0$. Its value is monotonically increased as $r \rightarrow 0$. In this case, $x_{s} \rightarrow 0$ and $y_{s} \rightarrow 0$ as well. Thus, taking into account 10 and (11), it is clear that

$$
\alpha_{\max }=\frac{1}{2} \operatorname{arctg} \frac{b}{2 d} .
$$

This formula shows that, if $d \rightarrow \infty$, then $\alpha_{\max } \rightarrow 0$.

Consider a typical situation when a sonar system is reasonably close to an object. Assuming that $d=0.5 \mathrm{~m}$ and the distance between sonars is $b=0.1 \mathrm{~m}$, we can find that $\alpha_{\max }=2.9^{\circ}$. For a more typical case, while $d=1 \mathrm{~m}$, the value of the angle $\alpha_{\max }$ is reduced down to $1.4^{\circ}$. As the angle is small, this can be used to approximate $\sin \alpha \sim$ $\alpha$ and $\cos \alpha \sim 1$. In this way, Eqn. (12) yields $\alpha$ as a function of $r$,

$$
\alpha=\frac{b}{4 d+r \sqrt{2}} .
$$

Keeping in mind that $\alpha$ is small, it is possible to approximate $x_{s}$ and $y_{s}$ as follows:

$$
x_{s}=g(r, d, b), \quad y_{s}=-g(r, d, b),
$$

where

$$
g(r, d, b)=\frac{r b}{2(2 \sqrt{2} d+r)} .
$$

Taking into account (8), (9) and (13), and then applying them to (8), the length of the signal path from sonar 1 to 2 can be determined. Then, using the obtained result and formula (6), we can substitute the appropriate terms in (4). Accordingly,

$$
\begin{aligned}
\rho=\frac{4}{b^{2}} & (g(r, d, b) \\
& \left.+\sqrt{\left(g(r, d, b)-\frac{b}{2}\right)^{2}+(g(r, d, b)-d)^{2}}\right)^{2} \\
& -1-\frac{4 d^{2}}{b^{2}} .
\end{aligned}
$$

This formula defines $\rho$ as an intricate function of parameters $b, d$ and $r$. Let us denote the function as $\rho_{c^{-} r}(r, d, b)$. Because $\rho$ for a rod is 0 and for a corner it is -1 and these values are independent of $b$ and $d$, it is 
necessary to check the form of the function for $r=0$ and $r \rightarrow \infty$, respectively. The first case can be easily investigated by direct substitution for $r$ in (14). It gives

$$
\rho_{c-r}(0, d, b)=\frac{4}{b^{2}}\left(\left(\sqrt{\frac{b^{2}}{4}+d^{2}}\right)^{2}-\frac{b^{2}}{4}-d^{2}\right)=0 .
$$

In the second case for $d \geq b / 2$, we find that

$$
\lim _{r \rightarrow \infty} \rho_{c-r}(r, d, b)=\frac{4}{b^{2}}\left(\left(\frac{b}{2}+d-\frac{b}{2}\right)^{2}-\frac{b^{2}}{4}-d^{2}\right)=-1 .
$$

This proves that the function meets the requirements mentioned above. For $d<b / 2$, the result is different. This is because it means that the sonars are inside a cylinder. That is, however, incompatible with the assumption that the sonar system is outside of the object.

\subsection{Transition from the corner to the rod and next to} the plane. In the previous sections, the functions $\rho_{c-r}$ and $\rho_{r-p}$ were constructed. Their images cover ranges $(-1,0]$ and $[0,1)$, respectively. For $r \rightarrow \infty$, the value -1 is the limit for $\rho_{c-r}$. Considering $\rho_{r-p}$ for the same condition, its limit is 1 . In order to obtain a function which represents a continuous transaction from the corner to the rod and then to the plane, both functions $\rho_{c-r}$ and $\rho_{r-p}$ can be combined in the following way:

$$
\rho_{c-p}(r, d, b)= \begin{cases}\rho_{c-r}(-r, d, b), & r \in(-\infty, 0), \\ \rho_{r-p}(r, d, b), & r \in[0,+\infty) .\end{cases}
$$

An example of this function drawn for $b=0.1 \mathrm{~m}$ and $d=1 \mathrm{~m}$ is shown in Fig. 16. It is interesting to find an

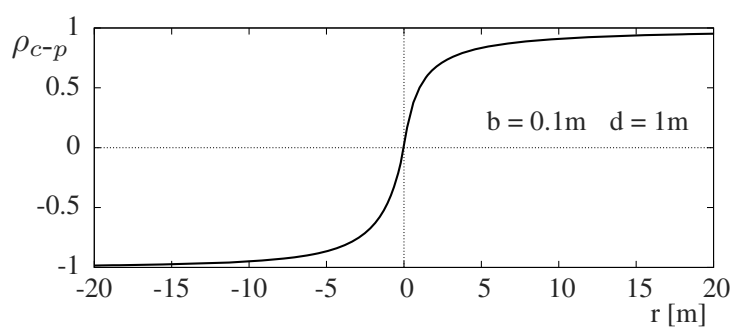

Fig. 16. Graph of the function $\rho_{c-p}$ for $b=0.1 \mathrm{~m}$ and $d=1$ $\mathrm{m}$.

answer to the question: Is the transition from the corner to the rod and then from the rod to the plane smooth or not? If so, it would suggest that this transition is natural. In order to check that, it is sufficient to determine the left limit of $\delta \rho_{c-r} / \delta_{r}$ for $r \rightarrow 0_{-}$and the right limit of $\delta \rho_{r-p} / \delta_{r}$ for $r \rightarrow 0_{+}$. Computing the derivatives, the following equations are obtained:

$$
\begin{aligned}
\lim _{r \rightarrow 0_{-}} \frac{\partial \rho_{c^{-}}}{\partial r}(r, d, b) & \\
= & -\frac{\partial \rho_{c^{-} r}}{\partial r}(0, d, b) \\
& =-\frac{\sqrt{2}}{d b}\left(\sqrt{\left(\frac{b}{2}\right)^{2}+d^{2}}-\left(\frac{b}{2}+d\right)\right),
\end{aligned}
$$

and respectively

$$
\begin{aligned}
\lim _{r \rightarrow 0_{+}} \frac{\partial \rho_{c-p}}{\partial r}(r, d, b) \\
\quad=\frac{\partial \rho_{r-p}}{\partial r}(0, d, b) \\
\quad=\frac{8 d}{b^{2}}\left(\sqrt{\frac{b^{2}}{4 d^{2}}+1}-1\right) .
\end{aligned}
$$

Both the obtained limits are different. Thus the combined transition, taking into account all cases considered, is not smooth.

\section{Conclusions}

In Section 5.1, the function $\rho_{r-p}(r, d, b)$ was determined, which allows $\rho$ to be computed for any stage of transition from the rod to the plane. The analogical function $\rho_{c^{-} r}(r, d, b)$ was determined for the transition from the corner to the rod in Section 5.2 These functions show that $\rho$ is the function of $r, d$ and $b$. In order to conclude that $\rho$ represents a feature of an object, it should be a function of object parameters for all possible transitions. The transition considered shows that it is not like that. The functions $\rho_{r-p}$ and $\rho_{c-r}$ depend on the gap size between sonars $b$ and the distance $d$ of the sonar system to an object. Thus, it proves that $\rho$ does not represent any feature of an object. Only in the three cases which represent a plane, a rod and a corner, are these functions reduced to a constant number. Therefore, in this situation the number directly represents an object.

It is beyond expectation that this parameter does not include any new information about an object. The same can be said about the proposed transition from a corner to a rod and next to a plane that is not smooth, which was shown in Section 5.3. Fortunately, this is good news because the last mentioned feature means that there are two phenomena described by two distinct models. The first one describes the phenomenon of signal reflection for a corner and any transitional form between a corner and a rod. The second one describes the phenomena of signal reflection for a plane and any transitional form between a plane and a rod. A question arises about what these phenomena are. Analyzing the case of a plane and the case of a corner, it can be noticed that the main difference is the number of signal reflections. For a plane, a signal 
is reflected from the plane's surface and directly goes to a receiver. For a corner, the signal is reflected twice. From thence it seems that these phenomena are single or double reflections. Unfortunately, the cases can be more complicated. For the situation presented in Fig. 17, it is

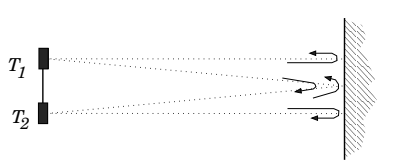

(a)

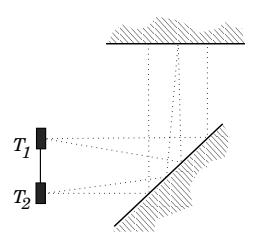

(b)
Fig. 17. Additional plane does not change the relations between lengths of signal paths reflected by the final plane.

possible to find such a case for a plane (see Fig. 17 a)) that, after introducing an additional plane, the lengths of signal paths are the same in both cases (see Fig. 17b)). This time, however, the signal is reflected two more times. A similar arrangement of objects can be constructed for a corner (see Fig. 18). The length of the path of the signal is the same in both cases (see Fig. 18 a),(b)) but the number of reflections is doubled (see Fig. 18b)). Thus it seems

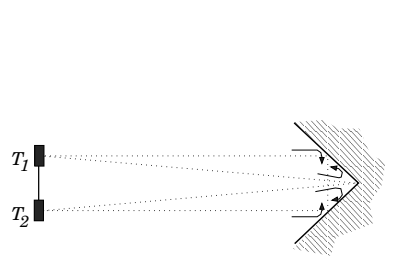

(a)

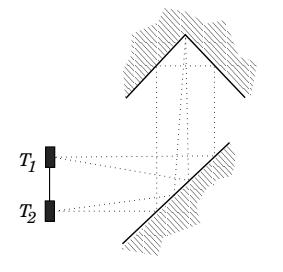

(b)
Fig. 18. Additional plane does not change the relations between lengths of signal paths reflected by a corner.

that the discussed phenomena are combined with an even or odd number of an reflections.

Finally, a hypothesis can be stated. In spite of the value of $\rho$ (excluding $-1,0$ and 1) not being combined with any object feature, the sign of this coefficient represents an object structure which causes an odd or an even number of reflections. It is worth investigating this problem more carefully. Its solution would make it possible to extract more information about objects in an environment. In this way, the objects could be distinguished more easily and used as natural landmarks for mobile robot navigation.

\section{References}

Adib, F. and Katabi, D. (2013). See through wall with Wi-Fi, ACM SIGCOMM'13, Hong Kong, China, pp. 75-86.

Barshan, B. (1999). Location and curvature estimation of spherical targets using multiple sonar time-of-flight measurements, IEEE Transactions on Instrumentation and Measurement 48(6): 1212-1223.

Barshan, B. and Kuc, R. (1990). Differentiating sonar reflections from corners and planes by employing an intelligent sensor, IEEE Transactions on Pattern Analysis and Machine Intelligence 12(6): 560 - 569.

Bozma, O. and Kuc, R. (1991). Building a sonar map in a specular environment using a single mobile sensor, IEEE Transactions on Pattern Analysis and Machine Intelligence 13(12): 1260-1269.

Brown, M.K. (1985). Feature extraction techniques for recognizing solid objects with an ultrasonic range sensor, IEEE Journal of Robotics and Automation 1(4): 191-205.

Heale, A. and Kleeman, L. (2001). Fast target classification using sonar, 2001 IEEE/RSJ International Conference on Intelligent Robots and Systems, Maui, HI, USA, Vol. 3, pp. 1446-1451.

Jackson, J.C., Summan, R., Dobie, G.I., Whiteley, S.M., Pierce, S.G. and Hayward, G. (2013). Time-of-flight measurement techniques for airborne ultrasonic ranging, IEEE Transactions on Ultrasonics, Ferroelectrics and Frequency Control 60(2): 343-355.

Kleeman, L. (2002). On-the-fly classifying sonar with accurate range and bearing estimation, IEEE/RSJ International Conference on Intelligent Robots and Systems, Lausanne, Switzerland, Vol. 1, pp. 178-183.

Kleeman, L. (2004). Advanced sonar with velocity compensation, International Journal of Robotics Research 23(2): 111-126.

Kleeman, L. and Kuc, R. (1994). An optimal sonar array for target localization and classification, IEEE International Conference on Robotics and Automation, San Diego, CA, USA, pp. 3130-3135.

Kleeman, L. and Kuc, R. (1995). Mobile robot sonar for target localization and classification, International Journal of Robotics Research 14(4): 295-318.

Kreczmer, B. (2010). Objects localization and differentiation using ultrasonic sensors, in H. Yussof (Ed.), Robot Localization and Map Building, InTech, Rijeka, pp. 521-543.

Kreczmer, B. (2013). Relations between classification criteria of objects recognizable by ultrasonic systems, 16th IEEE International Conference MMAR 2011, Międzyzdroje, Poland, pp. 806-811.

Kuc, R. and Siegel, M. (1987). Physically based simulation model for acoustic sensor robot navigation, IEEE Transactions on Pattern Analysis and Machine Intelligence PAMI9(6): 766-778.

Leonard, J.J. and Durrant-Whyte, H.F. (1991). Mobile robot localization by tracking geometric beacons, IEEE Transactions on Robotics and Automation 7(3): 376-382.

Leonard, J.J. and Durrant-Whyte, H.F. (1992). Directed Sonar Sensing for Mobile Robot Navigation, Kluwer Academic Publishers, Boston, MA/London/Dordrecht.

Möller, M.M. (1995). Autonomous mobility with triaural sonar system, International Symposium on Intelligent Robotic Systems, Pisa, Italy, pp. 25-30. 
Nanani, G.K. and Prasad, K.M.V.V. (2013). A study of wi-fi based system for moving object detection through the wall, International Journal of Computer Applications 79(7): 15-18.

Peremans, H., Audenaert, K. and Campenhout, J.M.V. (1993). A high-resolution sensor based on tri-aural perception, IEEE Transactions on Robotics and Automation 9(1): 36-48.

Peremans, H., Campengout, J.V. and Levrouw, L. (1991). Steps towards tri-aural perception, in P.S. Schenker (Ed.), Sensor Fusion IV: Control Paradigms and Data Structures, SPIE, Bellingham, WA, pp. 165-176.

Queirós, R., Corrêa Alegria, F., Silva Girão, P. and Cruz Serra, A. (2010). Cross-correlation and sine-fitting techniques for high-resolution ultrasonic ranging, IEEE Transactions on Instrumentation and Measurement 59(12): $1-10$.

Rencken, W.D., Peremans, H. and Möller, M. (1994). Tri-aural versus conventional sonar localisation and map building, JAS-4 Conference, Karlsruhe, Germany, pp. 398-402.
Bogdan Kreczmer received an M.Sc. degree in control engineering form the Wrocław University of Technology, Poland, in 1986, and an M.Sc. degree in computer science in 1988. He was granted a Ph.D. degree in robotics from the same university in 1998, where he currently works as an assistant professor at the Chair of Cybernetics and Robotics in the Department of Electronics. His research interests include ultrasonic sensing and mobile robot navigation.

Received: 29 October 2014 Revised: 18 May 2015

Re-revised: 7 August 2015 